\title{
Hybrid of Genetic Algorithm and Continuous Ant Colony Optimization for Optimum Solution
}

\author{
BAN A.MITRAS ${ }^{1}$ and ADEEBA KH.ABOO ${ }^{2}$ \\ ${ }^{1}$ Prof. Dr.Operation Research \& Artificial Techniques Department, College of Computer Science and \\ Mathematics, Mosul University, Mosul, Iraq \\ ${ }^{2}$ M. Sc. Student, Computer Science Department, College of Computer Science and Mathematics, Mosul \\ University, Mosul, Iraq \\ E-mail: 'dr.ban_mitras@yahoo.com,²dalyadiamond@yahoo.com
}

\begin{abstract}
This research proposes a hybrid approach by combining genetic algorithm (GA) and Continuous Ant Colony Optimization (CACO) to find optimum solutions, using a continuous ant colony algorithm as a mutation of genetic algorithm; the performance of the hybrid algorithm is illustrated using three test functions. The results show the efficiency and capabilities of the new hybrid algorithm in finding the optimum solutions.
\end{abstract}

Keywords: Genetic algorithm (GA), Continuous Ant Colony Optimization (CACO).

\section{INTRODUCTION}

The first evolutionary-based technique introduceed in the literature was the genetic algorithms (GAs), GAs were developed based on the Darwinian principle of the 'survival of the fittest' and the natural process of evolution through reproduction. Based on its demonstrated ability to reach nearoptimum solutions to large problems, the GAs technique has been used in many applications in science and engineering. Despite their benefits, GAs may require long processing time for a near optimum solution to evolve. Also, not all problems lend themselves well to a solution with Gas [1].

Ant Colony Optimization (ACO) was developed by Dorigo et al. Based on the fact that ants are able to find the shortest route between their nest and a source of food. This is done using pheromone trails, which ants deposit whenever they travel, as a form of indirect communication $[2,3]$.

As shown in Figure (1) when ants leave their nest to search for a food source, they randomly rotate around an obstacle, and initially the pheromone deposits will be the same for the right and left directions. When the ants in the shorter direction find a food source, they carry the food and start returning back, following their pheromone trails, and still depositing more pheromone. As indicated in Figure (1), an ant will most likely choose the shortest path when returning back to the nest with food as this path will have the most deposited pheromone. For the same reason, new ants that later starts out from the nest to find food will also choose the shortest path. Over time, this positive feedback (autocatalytic) process prompts all ants to choose the shorter path [1].

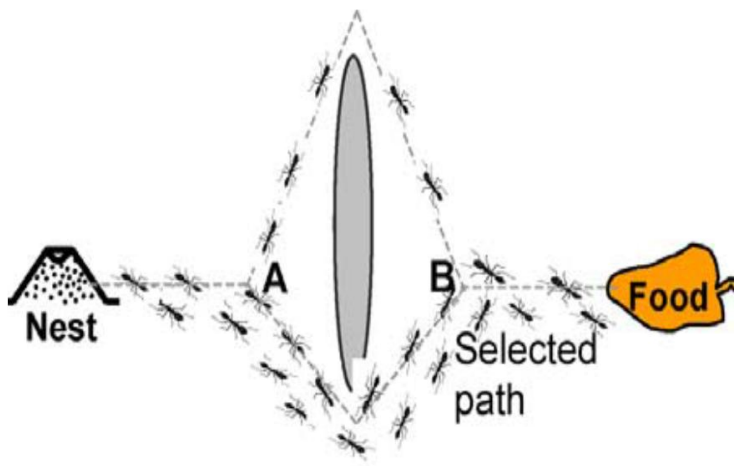

Fig. 1. Ant colony: Shortest path to food source 
B. A. Mitras and A. K. Aboo / International Journal of Computer Networks and Communications Security, 2 (1), January 2014

\section{GENETIC ALGORITHMS}

GAs is inspired by biological systems' improved fitness through evolution. A solution to a given problem is represented in the form of a string, called 'chromosome', consisting of a set of elements, called 'genes', that hold a set of values for the optimization variables. GAs work with a random population of solutions (chromosomes). The fitness of each chromosome is determined by evaluating it against an objective function. To simulate the natural survival of the fittest process, best chromosomes exchange information (through crossover or mutation) to produce offspring chromosomes. The offspring solutions are then evaluated and used to evolve the population if they provide better solutions than weak population members. Usually, the process is continued for a large number of generations to obtain a best-fit (near optimum) solution $[1,4]$.

\section{CONTINUOUS ANT COLONY OPTIMIZATION (CACO)}

CACO algorithm was a first attempt on the field of continuous optimization. It is suggested for a local search in the neighborhood of the place called nest. The CACO authors supposed to find this place by a global optimization method, for example Genetic Algorithm. The neighborhood is then searched by using vectors leading from the nest. Initially, they are uniformly distributed inside a global search radius and have a small amount of a pheromone [5]. Figure (2) illustrate the CACO initial state.

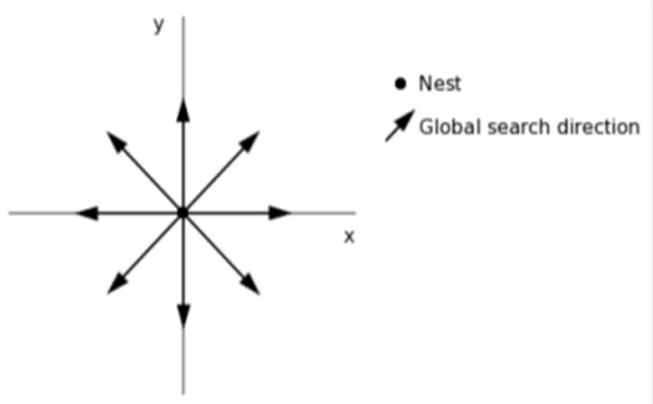

Fig. 2. CACO Initial State

Ant chooses direction using roulette wheel selection applied to the pheromone amounts. Than It moves randomly to the place the vector points and does a random walk inside a local search radius. This radius can shrink in time to do more detailed searches around the point. The ant Fitness is then calculated from its position and the amount of pheromone on choosing direction vector is proportionally increased. If a better solution is found, the vector is changed to actual ant position.

The pseudo-code for the CACO algorithm is outlined in Figure (3):

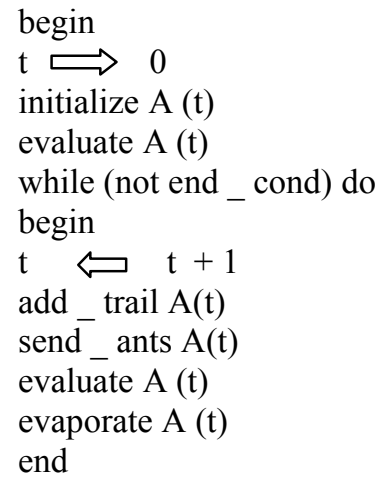

Fig. 3. pseudo code for the CACO

The function A (t) is the data structure representing the nest and its vicinity. The first step is to initialize the nest structure by generating random starting search direction vectors.

Next, the search radius is defined. This value determines the maximum distance that an ant can move at a single time. Then, "initialize A (t)" sends ants in various search directions while "evaluate A(t)" calls the objective function evaluation. The command "add_trail" is synonymous to the ants laying pheromones on the trails. This is the basic version of the CACO algorithm $[5,6,7]$.

\section{PROPOSED METHOD}

In this phase, the CACO is used as a mutation of $\mathrm{GA}$, the output of the GA is given as an input to the CACO. The genetic algorithm undergoes the selection, crossover process and it gives the result. The result contains only one value which is optimal value. This is the process going to proceed in our paper.

Steps of hybrid algorithm:

1. Initial population: Genetic algorithm begin its work with a group of chromo-somes (solutions) known as the initial population, members of this population be configured up randomly.

2. Fitness Function: This step is the evalua-tion of solutions (chromosome). 
B. A. Mitras and A. K. Aboo / International Journal of Computer Networks and Communications Security, 2 (1), January 2014

\section{The process of configure of the new generations}

\section{a. Selection}

There are many different selection methods, such as elitist selection, rank selection and roulette wheel selection. In this paper, the roulette wheel selection method is used. In roulette wheel selection, the individual is selected based on the relative fitness with its competitors. This is similar to dividing the wheel into a number of slices.

\section{b. Crossover}

There are many different crossover methods, in this paper, the arithmetic crossover is used. Arithmetic crossover operator linearly combines two parent chromosome vectors to produce two new offspring according to the equations:

Offspring $1=\mathrm{a} *$ Parent $1+(1-\mathrm{a}) *$ Parent 2

Offspring $2=(1-\mathrm{a}) *$ Parent $1+\mathrm{a} *$ Parent2

Where is a random weighting factor chosen before each crossover operation.

c. Using CACO as a mutation for GA according to the following steps:

1. 1.Chromosomes that have been obtained from the process of mating (crossover) is entered into ant colony algorithm, is calculate the probability(Pij) of each chromosome according to the equation Probability:

$$
P_{i j}=\frac{T_{i j}}{\sum_{j=1}^{p} T_{i j}}
$$

Where $T_{i j}$ is a amounts of pheromone, $T_{i j}=1$ for each chromosome (solution) in initial state of algorithm, $P$ number of chromosome, Set the iteration number $\mathrm{L}=1$.

2. Generate random numbers in the range $(0$, 1), one for each ant.

Number of ants $=$ number of chromosomes

3. Each ant chooses specific value (specific chromosome) if the ant be within the range of probability of the value (chromosome), this step is repeat for each ant.

4. Evaluate the objective function values; determine the best and worst values among the values chosen by different ants.

Fbest $=\min ($ values $)$

fworst $=\max$ (values)

The best value is stored during each iteration within a matrix.

5. Test for the convergence of the process. The process is assumed to converge if all ants take the same best value. If convergence is not achieved, assume that all the ants return home and start again in search of food. Set the new iteration number as $\mathrm{L}=\mathrm{L}+1$, and update the pheromones on different values as

$$
T_{i j}^{(L)}=T_{i j}^{\left({ }^{(o l d)}\right.}+\sum_{k} \Delta T^{(k)}
$$

Where $T_{i j}^{(\text {old })}$ denotes the pheromone amount of the previous iteration left after evaporation, which is taken as

$$
T_{i j}^{\left({ }^{\text {old })}\right.}=(1-P P) * T_{i j}^{(L-1)}
$$

and $\sum_{k} \Delta T^{(k)}$ is the pheromone deposited by the best ant $\mathrm{k}$ and the summation extends over all the best ants $\mathrm{k}$ (if multiple ants take the same best path). The evaporation rate or pheromone decay factor PP is assumed to be in the range 0.5 to 0.8 and the pheromone deposited $\sum_{k} \Delta T^{(k)}$ is computed using:

$$
\sum_{k} \Delta T^{(k)}=\frac{\text { fbest }}{\text { fworst }}
$$

With the new values of $T_{i j}{ }^{(L)}$, go to step 1. Steps $1,2,3,4$ and 5 are repeated until the process converges, that is, until all the ants choose the same best path.

In some cases, the iterative process is stopped after completing a pre-specified maximum number of iterations ( $\mathrm{L} \max )$. 
B. A. Mitras and A. K. Aboo / International Journal of Computer Networks and Communications Security, 2 (1), January 2014

6. Updated generation: The matrix contains that best value is enter to genetic algorithm as population.

7. Stop Criterion: The iterative process is stopped after completing a pre-specified maximum number of generations of genetic algorithm.

Figure (4) illustrate the proposed algorithm:

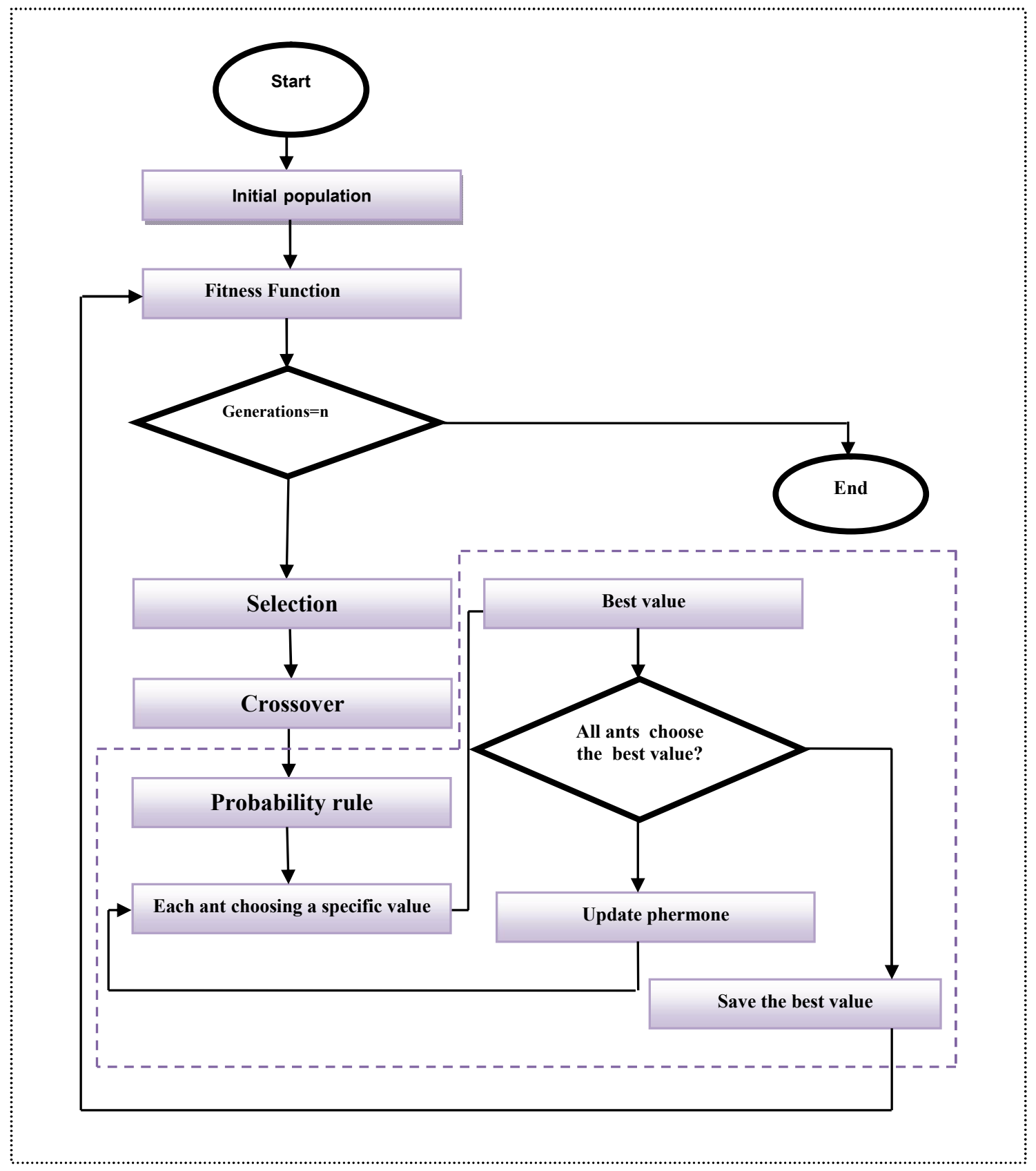

Fig. 4. The proposed algorithm 
B. A. Mitras and A. K. Aboo / International Journal of Computer Networks and Communications Security, 2 (1), January 2014

\section{NUMERICAL}

The proposed GA-CACO is tested using 3 benchmark functions. For comparison, GA, CACO, Table 1 shows the details of test functions.

Table 1: Benchmark functions (F1-F3)

\begin{tabular}{|l|l|c|l|}
\hline Benchmarks & Function & Range & Minimum \\
\hline F1 (Sphere) & $\sum_{i=1}^{n} x_{i}^{2}$ & {$[-5.12,5.12]$} & $f(0)=0$ \\
\hline F2 (Rastrigin) & $\sum_{i=1}^{n}\left(x_{i}^{2}-10 \cos \left(2 \pi x_{i}\right)+10\right)$ & {$[-5.12,5.12]$} & $f(0)=0$ \\
\hline F3 (Griewank) & $1+\sum_{i=1}^{n}\left(\frac{x_{i}^{2}}{4000}\right)-\prod_{i=1}^{n}\left(\cos \left(\frac{x_{i}}{\sqrt{i}}\right)\right)$ & {$[-600,600]$} & $f(0)=0$ \\
\hline
\end{tabular}

In Table 2, the performance of the GA-CACO algorithm is compared with GA.

Table 2: The results achieved by GA and GA-CACO onF1-F3

\begin{tabular}{|c|c|c|l|}
\hline Function & Generations & GA & \multicolumn{1}{|c|}{$\begin{array}{c}\text { GA- } \\
\text { CACO }\end{array}$} \\
\hline \multirow{4}{*}{ F1 } & 10 & $\begin{array}{l}1.6049 \mathrm{E}- \\
011\end{array}$ & $\begin{array}{l}2.6129 \mathrm{E}- \\
013\end{array}$ \\
\cline { 2 - 4 } & 20 & $1.0010 \mathrm{E}-$ & $1.5909 \mathrm{E}-$ \\
& & 010 & 13 \\
\cline { 2 - 4 } & & $1.9607 \mathrm{E}-$ & $6.1247 \mathrm{E}-$ \\
& 30 & 010 & 014 \\
\hline \multirow{4}{*}{ F2 } & 10 & $1.3840 \mathrm{E}-$ & $2.9280 \mathrm{E}-$ \\
& & 009 & 011 \\
\cline { 2 - 4 } & 20 & $1.9859 \mathrm{E}-$ & $3.2603 \mathrm{E}-$ \\
& & 008 & 011 \\
\cline { 2 - 4 } & 30 & $3.8898 \mathrm{E}-$ & $4.4409 \mathrm{E}-$ \\
& & 008 & 013 \\
\hline \multirow{3}{*}{ F3 } & 10 & $8.0091 \mathrm{E}-$ & $2.7756 \mathrm{E}-$ \\
& & 012 & 015 \\
\cline { 2 - 4 } & 20 & $5.0050 \mathrm{E}-$ & $1.0214 \mathrm{E}-$ \\
& & 011 & 014 \\
\hline
\end{tabular}


B. A. Mitras and A. K. Aboo / International Journal of Computer Networks and Communications Security, 2 (1), January 2014

\section{CONCLUSION}

This paper proposes a novel hybrid approach consisting GA and CACO and its performance is evaluated using various test functions. It illustrates the performance of the proposed hybrid approach using a set of well-known benchmark functions. The simulations indicate that the proposed algorithm has outstanding performance in speed of convergence and precision of the solution for global optimization. The results show the efficiency and capabilities of the new hybrid algorithm in finding the optimum.

\section{REFERENCES}

[1] Elbeltagi, E., Hegazy, T., Grierson, D.,(2005)," Comparison among five evolutionary-based optimization algorithms ", Advanced Engineering Informatics.

[2] Gao, S., Zhang, Z., and Cao. C.,(2010)," A Novel Ant Colony Genetic Hybrid Algorithm", Journal of Software, Vol. 5.

[3] Aravindh, S. and Michael, G.,(2012)," Hybrid of Ant Colony Optimization and Genetic Algorithm for Shortest Path in Wireless Mesh Networks ", Journal of Global Research in Computer Science, Vol. 3.

[4] Sivanaudom, S.N., Deepa, S.N. (2008), "An Introduction To Genetic Algorithms ", Springer - Verlag Berlin Heidelberg, India.

[5] Aidov, A., Dulikravich, G. S., (2009)," Modified Continuous Ant Colony Algorithm ", 2nd International Congress of Serbian Society of Mechanics.

[6] Kovarı, O., (2006), "Ant Colony Optimization for Continuous Problems", Msc. Thesis, Dept. of Electrical Engineering, University of Czech Technical.

[7] S., (2009), "Engineering Optimization: Theory and Practice", Fourth Edition, John Wiley \& Sons,Inc, publication, Canda.Feigen , L.P Physical characteristics of sound and hearing. American journal of Cardiology, 28 (2), 130 133, 1971. 